an alternative GRC site, but did this modification result in anxiolytic rather than anxiety-inducing effects?

In two rodent models of anxiety, treatment with compound 4 relieved anxiety-related behaviour. Notably, the magnitude of this effect was comparable to that afforded by diazepam. But the real breakthrough came from assessments of motor performance using the rotarod test: diazepam caused motor impairment whereas compound 4 did not, even at the maximal soluble dose. Compound 4 might therefore be at the forefront of a new approach to the design of robust anxiolytics with improved side-effect profiles.

Suzanne Farley

\section{(4) References and links} ORIGINAL RESEARCH PAPER

Johnstone, T. B. C. et al. Modifying quinolone antibiotics yields new anxiolytics. Nature Med. 30 Nov 2003 (doi: 10.1038/nm967) FURTHER READING Christopoulos, A. Allosteric binding sites on cell-surface receptors: novel targets for drug discovery. Nature Rev. Drug Discov. 1, 198-210 (2002)
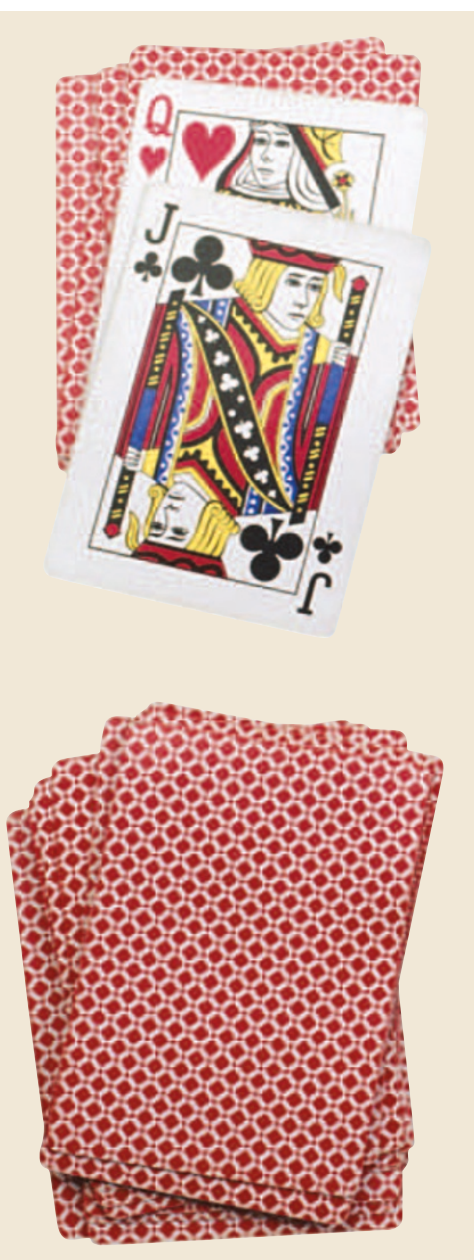

TARGET VALIDATION

\title{
Of mice and men and rats...
}

Knockout mice are now widely used to provide validation of the therapeutic potential of molecular targets - a strategy that is backed up by evidence that the knockout phenotypes for the targets of many leading drugs correlate well with known drug efficacy. Nevertheless, it is important to remember that this might not always be the case, as highlighted by a recent paper by Hirst et al. in Molecular Pharmacology, which demonstrates marked differences between the distribution and pharmacology of a particular serotonin receptor subtype in humans and rats, and that observed in mice.

Serotonin (5-hydroxytryptamine, 5-HT) is a neurotransmitter that exerts a wide variety of physiological and behavioural effects by acting on one or more of at least seven 5-HT receptor subtypes. The rat and human versions of the 5-HT ${ }_{6}$ receptor, which is a G-protein-coupled receptor, were cloned in the mid-1990s, and seem to show similar pharmacological properties and distribution in the brain. Since then, various studies have provided evidence that antagonists of the $5 \mathrm{HT}_{6}$ receptor might boost cholinergic neurotransmission and thereby ameliorate the cognitive impairments experienced by patients with dementia or schizophrenia, raising interest in this receptor as a drug target.

However, when Hirst and colleagues attempted to study the brain distribution of mouse 5- $\mathrm{HT}_{6}$ receptors using a radioligand known as $\left[{ }^{125} \mathrm{I}\right] \mathrm{SB}-258585$ that selectively labels the human and rat $5-\mathrm{HT}_{6}$ receptors, their experiments indicated that there was no specific binding of the radioligand. Prompted by these unexpected observations, they set out to compare the brain distribution of the $5-\mathrm{HT}_{6}$ receptor in mouse, rat and human brain by using the reversetranscriptase polymerase chain reaction. Indeed, they found that in the rat and human brain, $5-\mathrm{HT}_{6}$ receptors were expressed in several regions, particularly the striatum, but in the mouse there was far less expression of the 5- $\mathrm{HT}_{6}$ receptor in all brain regions examined, and no enrichment in the striatum.

The authors also carried out experiments to investigate the pharmacology of the $5-\mathrm{HT}_{6}$ receptor in mouse, rat and man using recombinant receptors. Binding studies showed that the affinity of a range of serotonergic agonists and antagonists at the mouse $5-\mathrm{HT}_{6}$ receptor differed considerably from that at the rat and human 5- $\mathrm{HT}_{6}$ receptors. Investigation of four amino acids identified from sequence alignments as potentially responsible for these differences using mutant $5-\mathrm{HT}_{6}$ receptors found that two residues - one in helix 5 and one in helix 6 accounted for the majority of the differences in pharmacology. Furthermore, computational studies of the receptor and ligands, which predicted that the binding pocket of the mouse $5-\mathrm{HT}_{6}$ receptor was different from the rat and human 5- $\mathrm{HT}_{6}$ receptors, were in excellent agreement with the results obtained from the experiments with the mutant receptors.

Overall, the findings of Hirst et al. show that the mouse $5-\mathrm{HT}_{6}$ receptor differs in many key respects from the $5-\mathrm{HT}_{6}$ receptor in rats and humans. In general, this suggests that before the phenotype of a mouse with a particular molecular target knocked out is accepted as validation of that target, or as a model of human disease, it would be wise to ascertain that the molecular target has a pharmacology and distribution in mice similar to that in humans.

Peter Kirkpatrick

(9) References and links

ORIGINAL RESEARCH PAPER Hirst, W. D. et al. Differences in the central nervous system distribution and pharmacology of the mouse 5-hydroxytryptamine-6 receptor compared with rat and human receptors investigated by radioligand binding, site-directed mutagenesis, and molecular modelling. Mol. Pharmacol. 64, 1295-1308 (2003)

FURTHER READING Zambrowicz, B. P. \& Sands, A. T. Knockouts model the 100 best-selling drugs - will they model the next 100 ? Nature Rev. Drug Discov. 2, 38-51 (2003)

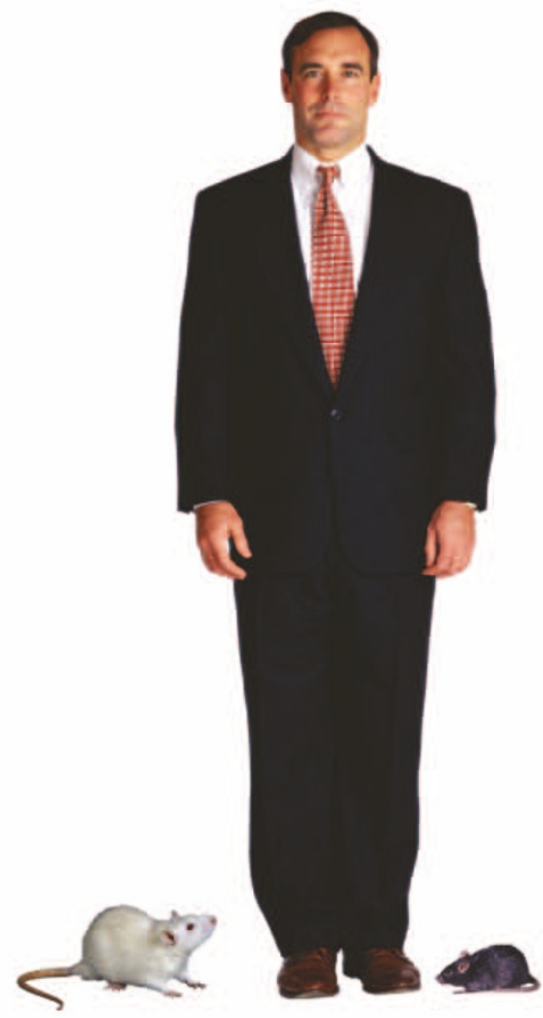

